Research Article

\title{
The Mechanisms of Improving IVF Outcomes of Liu-Wei-Di-Huang Pill Acting on DOR Patients
}

\author{
Jimei Xiao, ${ }^{1}$ Jingyan Song, ${ }^{1}$ Yuanhong Sa, ${ }^{1}$ Lihua Yuan, ${ }^{1}$ Jiayin Guo, ${ }^{2}$ and Zhengao Sun $\mathbb{D}^{1,3}$ \\ ${ }^{1}$ The First Clinical College, Shandong University of Traditional Chinese Medicine, Jinan 250014, China \\ ${ }^{2}$ Guangdong Provincial Key Laboratory of New Drug Screening, School of Pharmaceutical Sciences, Southern Medical University, \\ Guangzhou 510515, China \\ ${ }^{3}$ Reproductive and Genetic Center of Integrated Traditional and Western Medicine, \\ The Affiliated Hospital of Shandong University of Traditional Chinese Medicine, Jinan 250011, China
}

Correspondence should be addressed to Zhengao Sun; sunzhengao77@126.com

Received 8 March 2020; Revised 26 July 2020; Accepted 19 October 2020; Published 31 October 2020

Academic Editor: Siyaram Pandey

Copyright (c) 2020 Jimei Xiao et al. This is an open access article distributed under the Creative Commons Attribution License, which permits unrestricted use, distribution, and reproduction in any medium, provided the original work is properly cited.

Diminished ovarian reserve (DOR) is the weakening of ovarian oocyte production and quality. It will further become premature ovarian failure without timely cure. However, disease pathology and diagnostic markers are still incompletely understood. LiuWei-Di-Huang (LWDH) pill, a traditional Chinese medicine formula, is commonly used in the treatment of DOR in China. To explore the mechanism of the effect of LWDH on in vitro fertilization (IVF) outcomes in patients with DOR, a pseudotargeted metabolomics study combined with multivariate data processing strategy was carried out. A liquid chromatography tandem mass spectrometry-based metabolomics approach was applied to characterize metabolic biomarker candidates. Multiple pattern recognition was used to determine groups and confirm important variables. A total of 21 potential biomarkers were characterized, and related metabolic pathways were identified. The study displayed that the established pseudotargeted metabolomics strategy is a powerful approach for investigating the mechanism of DOR and LWDH. In addition, the approach may highlight biomarkers and metabolic pathways and can capture subtle metabolite changes from headache, which may lead to an improved mechanism understanding of DOR diseases and LWDH treatment.

\section{Introduction}

Diminished ovarian reserve (DOR) is often found in the process of long-term infertility seeking assisted pregnancy, which affects female sex hormone levels and fertility [1]. Previous studies have found that the incidence of DOR is gradually increasing and showing a younger trend [2]. In assisted reproductive technology, ovarian reserve has become an important factor affecting the outcome of assisted reproductive technology (ART). Liu-Wei-Di-Huang (LWDH) pill is a traditional Chinese medicine (TCM) formula for the treatment of DOR, with high efficiency and low toxicity in China [3]. It provides a good clinical effect in restoring menstrual cycle, protecting ovarian function in advance, and improving ART outcomes [4]. Xia and Wang improved ovarian function in patients with DOR by reestablishing yin-yang balance of heart-kidney-uterus axis and restoring normal reproductive endocrine using $\mathrm{LWDH}$ [5]. However, the specific mechanism is not yet clear.

Follicular fluid is a product of secretion of granulosa cells and testicular cells through the serum follicular barrier to provide nutrients for oocyte development [6]. The change in follicular fluid microenvironment will affect the occurrence and quality of oocytes [7]. At present, metabolomics as a powerful analytical strategy is applied in many fields such as biomarker discovery and TCM study $[8,9]$, which could provide the actual representation of the system [10]. Liquid chromatography tandem mass spectrometry (LC-MS) has become a preferred strategy in metabolomics study [11]. LCMS mainly includes triple quadrupole mass spectrometry $(\mathrm{QqQ})$, triple quadrupole-linear ion trap mass spectrometry (QTRAP), quadrupole-time of flight mass spectrometry 
(QTOF), and orbitrap mass spectrometry. QqQ or QTRAP could provide the analytical sequence stability and repeatability [12]. Despite orbitrap has a higher resolution rate, the QTOF analyzer possesses a much higher scanning rate, which is more suitable in the complex system and biofluids than orbitrap [13]. Therefore, QTOF- and QqQ-based pseudotargeted metabolomics method [14] was developed to characterize the metabolic biomarkers on SOR. Pseudotargeted metabolomics, taking into account the advantages of nontargeting metabolomics and targeting metabolomics, overcomes their respective shortcomings [15]. It could provide high-quality and rich-information data for metabolomics study [16]. A pattern recognition approach was carried out to estimate the changes in metabolite levels in follicular fluid and to identify 21 potential biomarkers of SOR. The integrated metabolomics study demonstrated that LWDH pill could regulate differently for different potential biomarkers. It may have more effective targets, with more comprehensive actions on the improvement of DOR. The mechanism of LWDH in treating DOR of kidney-yin deficiency was explored to provide scientific basis for clinical treatment.

\section{Materials and Methods}

2.1. Materials. HPLC-grade methanol, acetonitrile, water, ammonium acetate, and formic acid were all supplied by Fisher Scientific (Fair Lawn, NJ, USA). The internal standards of chloramphenicol and clenbuterol were from SigmaAldrich (St. Louis, MO, USA). In our study, $3.2 \mathrm{~kg}$ of prepared radix rehmanniae, $1.6 \mathrm{~kg}$ of prepared rhizoma dioscoreae, $1.6 \mathrm{~kg}$ of prepared fructus corni, $1.2 \mathrm{~kg}$ of prepared tuckahoe, $1.2 \mathrm{~kg}$ of prepared rhizoma alismatis, and $1.2 \mathrm{~kg}$ of prepared tree peony bark were crushed by pulverizer. Then, the refine honey was added to the herbal powder at a ratio of 3 to 10 . They were kneaded for half an hour and prepared into LWDH pills with a mold. LWDH pills were obtained from the Affiliated Hospital of Shandong University of Traditional Chinese Medicine (Jinan, China) and authenticated by Dr. Sun (Shandong University of Traditional Chinese Medicine, Jinan, China).

2.2. Subjects. For the pseudometabolomics analysis, the MetSizeR approach for sample size estimation was used to estimate a total sample size of 84 subjects using the following assumptions: 954 follicular fluid metabolites, a target false detection rate of $5 \%$, and an expected proportion of significant metabolites of $20 \%$ [17]. Subjects needed $(n=84)$ were recruited, and their follicular fluid was collected at the Affiliated Hospital of Shandong University of Traditional Chinese Medicine, from May 2018 to February 2019. For the purpose of this study, the subjects were divided into the pretreatment group $(n=28)$, the posttreatment group $(n=28)$, and the control group $(n=28)$. The diagnosis of DOR is done via expert consensus on hormone replacement therapy for premature ovarian insufficiency [18]. Subjects in the treatment group orally took one LWDH pill each time, twice a day, until the day of hCG trigger. The study was approved by the Health Authorities and Ethics Committees of the Affiliated Hospital of Shandong University of Traditional Chinese Medicine. All subjects signed the informed consent prior to being included in the study.

2.3. Inclusion and Exclusion Criteria. Inclusion criteria of the DOR patients are as follows: (1) kidney-yin deficiency of traditional Chinese medicine; (2) 20-40-year-old; (3) 10 IU/ $\mathrm{ml}<\mathrm{FSH}<25 \mathrm{IU} / \mathrm{ml}$; (4) patients of the control group who had a history of induced abortions of a normal pregnancy.

Inclusion criteria of the control group are as follows: (1) infertility due to male factor; (2) 20-40-year-old.

Exclusion criteria are as follows: (1) congenital genital dysplasia; (2) cerebrovascular and cardiovascular; (3) hyperprolactinemia and polycystic ovary syndrome; (4) fail to receive treatment as required due to personal and other reasons; (5) poor treatment effect.

2.4. Collection and Preparation of Follicular Fluid. Follicular fluid samples (approximately $1.0 \mathrm{~mL}$ ) were collected according to the previous protocol [19]. Follicular fluid samples of $100 \mu \mathrm{L}$ were mixed with $300 \mu \mathrm{L}$ of methanol containing $500 \mathrm{ng} / \mathrm{mL}$ of internal standards. The mixture was vortexed for $2 \mathrm{~min}$ and then centrifuged at $13000 \times g$ for $20 \mathrm{~min}$, at $4^{\circ} \mathrm{C}$. The supernatant was then transferred to an autosampler plate for analysis.

2.5. Mass Spectrometry Analysis. The separation was performed using a SCIEX ExionLC AD ultraperformance liquid chromatography system (SCIEX, CA, USA), while the chromatographic separation was performed on a Waters HILIC $(100 \mathrm{~mm} * 2.1 \mathrm{~mm}, 1.7 \mu \mathrm{m})$ column (Waters, Milford, MA, USA) at $40^{\circ} \mathrm{C}$. Analysis was completed with a gradient elution of $0.1 \%$ formic acid in acetonitrile $(A)$ and $10 \mathrm{mM}$ ammonium acetate in water (B) within $10.0 \mathrm{~min}$. The gradient program was $5 \% \mathrm{~B}$ at $0.0-0.3 \mathrm{~min}, 95 \% \mathrm{BA}$ at $5.0-8.0 \mathrm{~min}$, and $5 \% \mathrm{~B}$ at $8.1-10.0 \mathrm{~min}$ at a flow rate of $0.4 \mathrm{~mL} / \mathrm{min}$ with a sample injection volume of $10.0 \mu \mathrm{L}$. All the samples were kept at $4^{\circ} \mathrm{C}$ during the analysis.

The MS system was performed using a SCIEX QTRAP5500 (SCIEX, Redwood City, CA, USA). The multireaction monitor (MRM) and enhanced production (EPI) data were acquired in positive/negative electrospray ionization mode with dynamic background subtraction (DBS). The MRM transitions were extracted from the follicular fluid MS/MS spectra, which were acquired from TripleTOF5600 (SCIEX, Redwood City, CA, USA). Source parameters were defined as follows: temperature, $550^{\circ} \mathrm{C}$; ion spray voltage, $5500 \mathrm{~V}$ (positive)/-4500 V (negative); nebulizer gas (gas 1), 60 psi; heater gas (gas 2), 60 psi; curtain gas, 30 psi; and declustering potential, $60 \mathrm{~V}$. For IDA, any MRM survey scan peak exceeding $150 \mathrm{cps}$ was selected for dependent scan. 5 candidate ions were allowed per cycle. The collision energy was set to $35 \mathrm{~V}$ (positive)/-35 V (negative) with a collision energy spread of $15 \mathrm{~V}$. All operations and data acquisition were controlled by the Analyst 1.6 software (SCIEX, Redwood City, CA, USA). 
2.6. Data Processing and Multivariate Data Analysis. The LCMS data files were imported into MQ software (SCIEX, Redwood City, CA, USA). The chromatographic peaks were extracted, respectively, while Pareto scaling and multivariate analyses of log transformation were applied to the data processing before LPS-DA was performed to give the contribution of the metabolites by VIP score. All the compounds with significance threshold satisfying corrected $p$ value cutoff 0.05 in one-way ANOVA and VIP score $>1$ were considered as potential biomarkers. The software used for other statistical analysis was MetaboAnalyst software 4.0 (Xia Lab at McGill University, Montreal, QC, Canada).

2.7. Metabolic Pathway. OS software (SCIEX, Redwood City, CA, USA) was used to help confirm the potential differential metabolites by analyzing with the fragmentation patterns, chromatographic retention characteristics, and comparing the mass-spectra with the authentic standards. The fragmentation of the potential metabolites was matched with the metabolites from online databases HMDB (http:// www.hmdb.ca).

Pathway analysis using the KEGG pathway database was performed by MetaboAnalyst software for the identified potential metabolites with significant changes in identifying the top DOR-correlated metabolic pathways. Meanwhile, pathway topology analysis was used to generate the impact value of the relative metabolic pathways using a relativebetweenness centrality test.

\section{Results}

3.1. Clinical Background. There was no significant difference in age, infertility years, body mass index, basic LH, basic E2, among basic $p(p>0.05)$ among the posttreatment group, the pretreatment group, and the control group. The results are shown in Table 1 for details.

3.2. Syndrome Integral. The total syndrome integral of DOR patients decreased significantly after treatment using LWDH pill $(p<0.001)$ (seen in Table 2$)$. The syndrome including YaoXiSuanRuan, WuXinFanRe, KouGanYanZao, dizziness, emaciation, and forgetfulness was significantly improved after treatment $(p<0.05)$. It is suggested that LWDH pill can improve the syndrome of kidney-yin deficiency of DOR patients. The results are shown in Table 3.

3.3. Clinical Results. There was no significant difference in hCG daily endocrine between the pretreatment and posttreatment $(p>0.05)$. The number of oocytes $(<14 \mathrm{~mm}$ follicles or $\geq 14 \mathrm{~mm}$ follicles) was statistically different $(p<0.05)$ during the day of hCG between pretreatment and posttreatment, which indicated that LWDH pill could improve the ovarian reserve of DOR patients and effectively stimulate the growth of follicles. The results showed that there were significant differences between pretreatment and posttreatment in the number of transplantable embryos and IVF fertilization rate $(p<0.05)$. However, there was no significant difference in the level of E2, the level of $\mathrm{P}$, the number of high-quality embryos, the oocyte acquisition rate, and the clinical pregnancy rate $(p>0.05)$. The detail information is shown in Table 4 .

3.4. Multivariate Statistical Analysis of Metabolite Profiling. A large-scale multireaction monitor (MRM) was applied in the LC-MS detection. Typical chromatograms of follicular fluid are shown in Figure 1. In order to eliminate the complex matrix interference of follicular fluid and confirm them, their MS/MS spectrum was compared with the reference MS/MS spectrum. For example, the comparison spectrum of $N$-acetyltryptophan is shown in Figure 2. Chromatographic peaks were then carried out for alignment and normalization, followed by multivariate statistical analysis. Partial least-squares discriminant analysis (LPSDA), a supervised multivariate analysis comparing with principal component analysis (PCA), was employed for metabolomics study to differentiate among the groups. As shown in the PLS-DA score plots, follicular fluid samples from the control group, the pretreatment group, and the posttreatment group were well separated into three categories (Figure 3), suggesting that metabolic perturbation significantly occurred in DOR patients as well as the LWDH pill treatment. All significantly differentiated metabolites satisfying corrected $p$ value cutoff 0.05 in one-way ANOVA were listed. A total of 21 potential metabolic biomarkers were found after follicular fluid metabolomics screening. The results indicated that the level of testosterone, progesterone, phosphorylcholine, $p$-cresol sulfate, dihomolinoleic acid, decanoylcarnitine, pipecolic acid, 13,14-dihydroretinol, choline, acrylamide, isobutyryl-L-carnitine, indole, and 3-oxo-octadecanoic acid in follicular fluid were downregulated in DOR patients, and inversely, the level of $\mathrm{N}$-acetyltryptophan, 2-propenyl 1-(1-propenylsulfinyl)propyl disulfide, eicosatrienoic acid, $20 \alpha$-dihydroprogesterone, 17-beta-estradiol-3,17-beta-sulfate, tryptophan, lactate, oxaloacetate, and O-decanoyl-L-carnitine upregulated. After the treatment of LWDH, the level of all 21 biomarkers in follicular fluid significantly backregulated comparing with the pretreatment group. The contribution of each potential biomarker to the discrimination between the two groups was ranked as VIP scores shown in Figure 4. The heatmap (Figure 5) using MetaboAnalyst 4.0 demonstrated different distribution patterns of totally 21 potential biomarkers among the four groups, and moreover, the results of hierarchical cluster analysis (HCA) provided a distinct visualization of the groups [20].

3.5. Pathway Analysis and Metabolic Network. The metabolic pathways related to the identified potential biomarkers were estimated by pathway topology analysis using MetaboAnalyst based on the KEGG reference pathways, in order to find possible metabolic pathways that have great impact on DOR. A figure of metabolic pathway impact which demonstrated the pathway impact values is shown in Figure 6. The results indicated that steroid hormone biosynthesis, glycerophospholipid metabolism, citrate cycle, alanine, 
TABLE 1: Clinical background of subjects.

\begin{tabular}{|c|c|c|c|c|}
\hline Item & Posttreatment & Pretreatment & Control & $p$ \\
\hline Age & $34.75 \pm 3.47$ & $32.97 \pm 4.01$ & $31.14 \pm 3.38$ & $\begin{array}{c}> \\
0.05\end{array}$ \\
\hline $\begin{array}{l}\text { Infertility } \\
\text { years }\end{array}$ & $4.36 \pm 2.16$ & $3.66 \pm 1.72$ & $2.79 \pm 1.62$ & $\begin{array}{c}> \\
0.05\end{array}$ \\
\hline BMI & $22.17 \pm 1.70$ & $21.42 \pm 1.74$ & $21.63 \pm 1.34$ & $\begin{array}{c}> \\
0.05\end{array}$ \\
\hline Basic LH & $4.45 \pm 1.62$ & $4.69 \pm 1.82$ & $4.69 \pm 1.78$ & $\begin{array}{c}> \\
0.05\end{array}$ \\
\hline Basic E2 & $48.43 \pm 17.06$ & $40.99 \pm 20.43$ & $38.45 \pm 12.01$ & $\begin{array}{c}> \\
0.05\end{array}$ \\
\hline Basic P & $0.62 \pm 0.29$ & $0.74 \pm 0.24$ & $0.66 \pm 0.21$ & $\begin{array}{c}> \\
0.05\end{array}$ \\
\hline
\end{tabular}

TABLE 2: Total integral of pretreatment and posttreatment using LWDH pill.

\begin{tabular}{lcccc}
\hline Item & Total integral & Coeffective & $T$ & $P$ \\
\hline Pretreatment & $15.11 \pm 3.119$ & 0.765 & 13.811 & $<0.001^{*}$ \\
Posttreatment & $9.86 \pm 2.445$ & 0.765 & & \\
\hline
\end{tabular}

*The difference between the two groups was of detectable statistical significance.

aspartate and glutamate metabolism, and tryptophan metabolism would be considered closely related to DOR (impact $>0.01$ ). Consequently, based on the level of the backregulation on biomarkers after the LWDH treatment, these metabolic pathways were presumed as the pathways related to the treatment of LWDH for DOR.

\section{Discussion}

The clinical manifestations of DOR were including low menstrual volume, irregular menstruation, and infertility. Patients with DOR often have symptoms of deficiency of yin and essence, such as palpitation, sweating, irritability, insomnia, and forgetfulness. As the most basic essence of human life, the kidney stores essence. When it is sufficient, women can be gestated by menstruation. With the increase in age, kidney essence gradually decreased, and reproductive function declined. LWDH pill could fill lean marrow and nourish yin and kidney. The integral of the posttreatment group was significantly lower than that of the pretreatment group, seen in Table 2. In clinical, it was found that there were significant differences in the number of $<14 \mathrm{~mm}$ follicles and $\geq 14 \mathrm{~mm}$ follicles between the posttreatment group and the pretreatment group. It shows that LWDH pill can improve the clinical symptoms of DOR patients.

Choline is essential for acetylcholine biosynthesis. Acetylcholine is a key neurotransmitter for neuron differentiation and maturation. A large intake of choline during pregnancy is beneficial to the development of embryo and the cognitive function of offspring [21,22]. During pregnancy, there is a high demand for choline, and large intake of choline does not increase the content of choline in urine $[23,24]$. Choline is also the main dietary source of methyl and affects DNA methylation as a methyl donor, while DNA methylation is related to apoptosis $[25,26]$. Linoleic acid can be metabolized to other biological activities, such as arachidonic acid. It has been found that the concentration of linoleic acid in the dominant follicle is high, and the concentration of arachidonic acid is low [27]. Linolenic acid has a favorable effect on the maturation of goat oocytes in vitro, which can promote the development of embryos and reduce the expression of apoptosis genes [28]. 3-Oxo-octadecanoic acid is the intermediate of fatty acid biosynthesis. It may affect the oocyte quality by affecting fatty acid metabolism. After intervention of traditional Chinese medicine indicates, the content of 3-oxo-octadecanoic acid was upregulated near into the normal level. Carnitine is necessary for energy metabolism [29]. It will make active fatty acids into mitochondria, where they are oxidized and broken down. Carnitine can protect the continuous growth of oocytes and embryos and improve the quality of oocytes and the potential of embryo development [30] and pregnancy rate [31]. It promotes the maturation of porcine oocytes and the development of parthenogenetic embryos by accelerating nuclear maturation and preventing oxidative damage and apoptosis [32]. In vitro carnitine can improve the outcome of embryos in the culture medium for oocyte maturation and embryo growth [33]. Fathi et al. found that the rate of cleavage and embryo development was faster when L-carnitine was added during in vitro maturation (IVM), and the development rate of morula and blastocyst stages was higher [34]. You et al. found that carnitine during IVM of oocytes improved the developmental ability of SCNT embryos [35]. It may be that the increase in GSH synthesis in the recipient oocytes reduces ROS level and stimulates nuclear reprogramming by increasing the expression of Pou5f 1 and transcription factors. Some studies have shown that carnitine has a protective effect on the nephrotoxicity, and the possible mechanism is to inhibit the production of reactive oxygen species, lipid peroxidation, matrix remodeling, and apoptosis, as well as anti-inflammatory [36].

Pyruvic acid is a kind of weak organic acid. It is the intermediate substance of Chinese medicine for the synthesis and decomposition of many substances, including oxidation metabolism, gluconeogenesis pathway, tricarboxylic acid cycle, and lipid synthesis. Lactic acid is the metabolite of glycolysis, which is closely related to energy metabolism, lipid metabolism, protein metabolism, and glucose metabolism. It was found that glucose and pyruvate affect the cytoplasmic maturation of porcine oocytes through glycolysis. Pyruvate can improve ROS, GSH, ATP, and early embryonic development of MII oocytes and promote the cytoplasmic maturation of pigs by providing energy and reducing oxidative stress $[37,38]$. In our study, after the treatment of LWDH pill, lactate was downregulated near into the normal level in patients with DOR, which may have an impact on the quality of oocytes.

Testosterone is a kind of anabolic steroid hormone. It plays an important role in ovarian response through androgen receptor (AR) pathway and non-AR pathway. It can induce the activation of primordial follicles by inhibiting PTEN expression via the AR pathway [39]. The increase in AR expression can promote the proliferation of granulosa cells and inhibit apoptosis [40] and increase the content of 
TABLE 3: The integral of each syndrome.

\begin{tabular}{|c|c|c|c|c|c|}
\hline Item & Pretreatment & Posttreatment & Coeffective & $T$ & $P$ \\
\hline YaoXiSuanRuan & $3.21 \pm 0.787$ & $2.46 \pm 1.427$ & 0.403 & 3.000 & $0.006^{*}$ \\
\hline WuXinFanRe & $3.11 \pm 0.567$ & $1.29 \pm 1.512$ & 0.222 & 6.460 & $<0.001^{*}$ \\
\hline KouGanYanZao & $1.14 \pm 0.705$ & $0.64 \pm 0.678$ & 0.575 & 4.415 & $<0.001^{*}$ \\
\hline Dizziness & $1.18 \pm 0.819$ & $0.75 \pm 0.441$ & 0.231 & 2.714 & $0.011^{*}$ \\
\hline Emaciation & $0.32 \pm 0.476$ & $0.18 \pm 0.39$ & 0.677 & 2.121 & $0.043^{*}$ \\
\hline Forgetfulness & $0.43 \pm 0.69$ & $0.18 \pm 0.39$ & 0.668 & 2.553 & $0.017^{*}$ \\
\hline
\end{tabular}

*The difference between the two groups was of detectable statistical significance.

TABLe 4: The number of follicles and endocrine parameters during the day of hCG.

\begin{tabular}{|c|c|c|c|}
\hline Item & Posttreatment & Pretreatment & $P$ \\
\hline$<14 \mathrm{~mm}$ follicles & $3.75 \pm 1.624$ & $2.69 \pm 1.775$ & $0.022 *$ \\
\hline$\geq 14 \mathrm{~mm}$ follicles & $4.04 \pm 1.453$ & $3.14 \pm 1.552$ & $0.028^{*}$ \\
\hline $\mathrm{LH}$ & $7.23 \pm 4.16$ & $5.2 \pm 4.77$ & 0.093 \\
\hline E2 & $1187.71 \pm 712.681$ & $1143.52 \pm 700.787$ & 0.814 \\
\hline $\mathrm{P}$ & $1.17 \pm 0.62$ & $1.24 \pm 0.66$ & 0.681 \\
\hline Number of oocytes & $3.75 \pm 1.531$ & $2.72 \pm 1.412$ & $0.011^{*}$ \\
\hline Number of transplantable embryos & $1.68 \pm 1.124$ & $0.97 \pm 0.778$ & $0.007^{*}$ \\
\hline Number of high-quality embryos & $0.32 \pm 0.723$ & $0.21 \pm 0.412$ & 0.464 \\
\hline Oocyte acquisition rate $(\%)$ & $92.9 \%$ & $86.8 \%$ & 0.145 \\
\hline IVF fertilization rate $(\%)$ & $73.9 \%$ & $52.5 \%$ & $0.007^{*}$ \\
\hline Clinical pregnancy rate $(\%)$ & $25 \%$ & $20.7 \%$ & 0.698 \\
\hline
\end{tabular}

*The difference between the two groups was of detectable statistical significance.

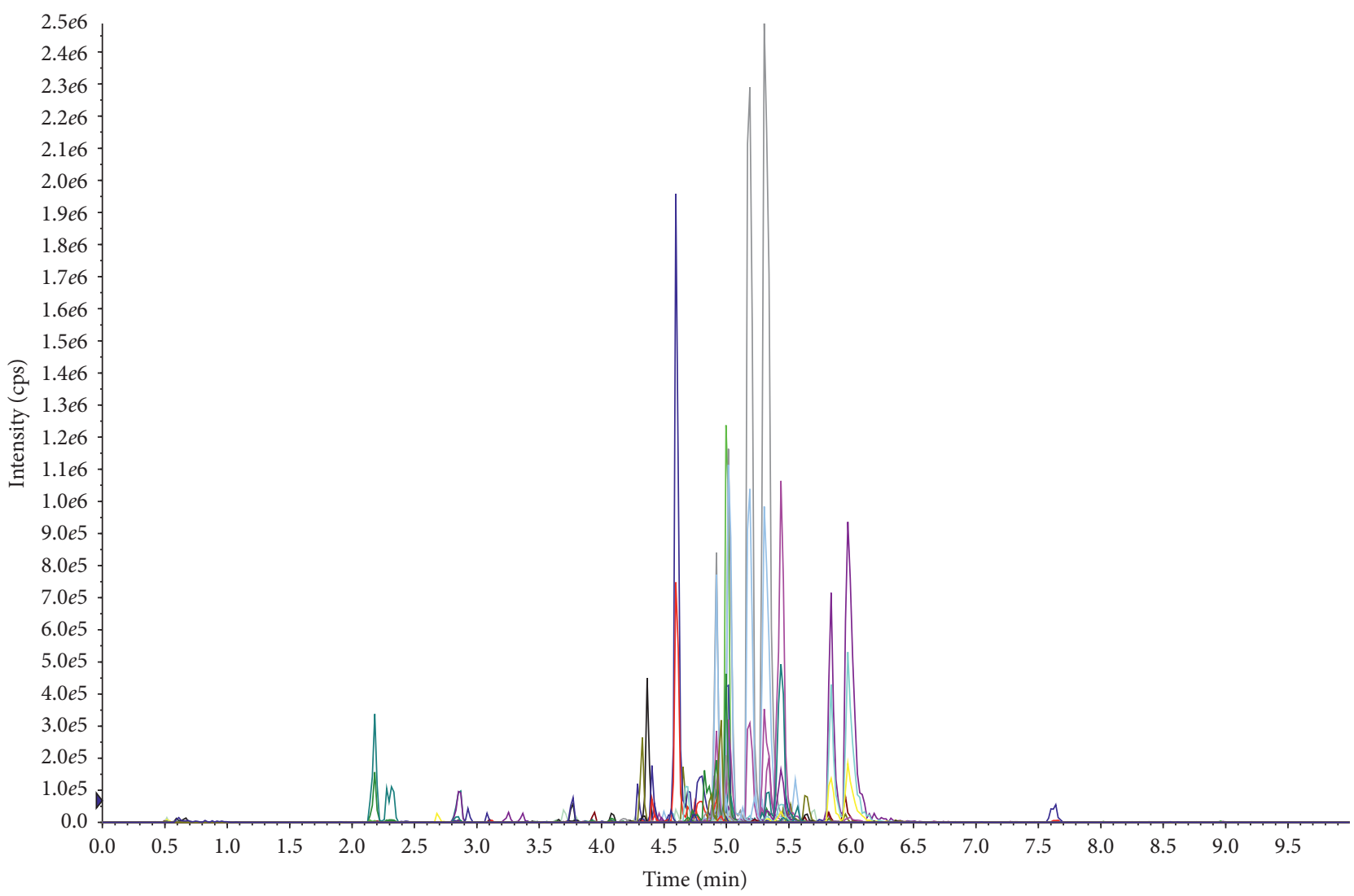

FIgURE 1: The typical chromatograms of follicular fluid samples.

insulin-like growth factor-1 (IGF-1) and growth differentiation factor-9 (GDF-9) in follicular fluid. It plays a role in follicle development and oocyte quality [41]. It can enhance the expression of follicle-stimulating hormone receptor (FSHR) in granulosa cells, improve the follicular sensitivity of FSH in the antral follicular phase, and increase the 


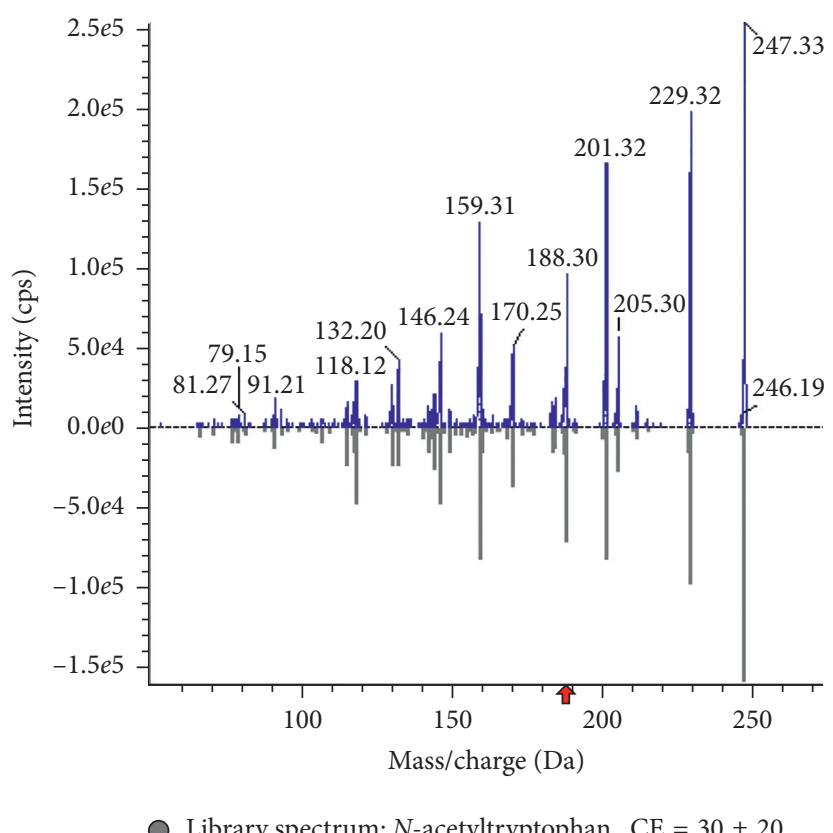

FIGURE 2: The comparison spectrum of $N$-acetyltryptophan between the experimental and reference MS/MS spectrum.

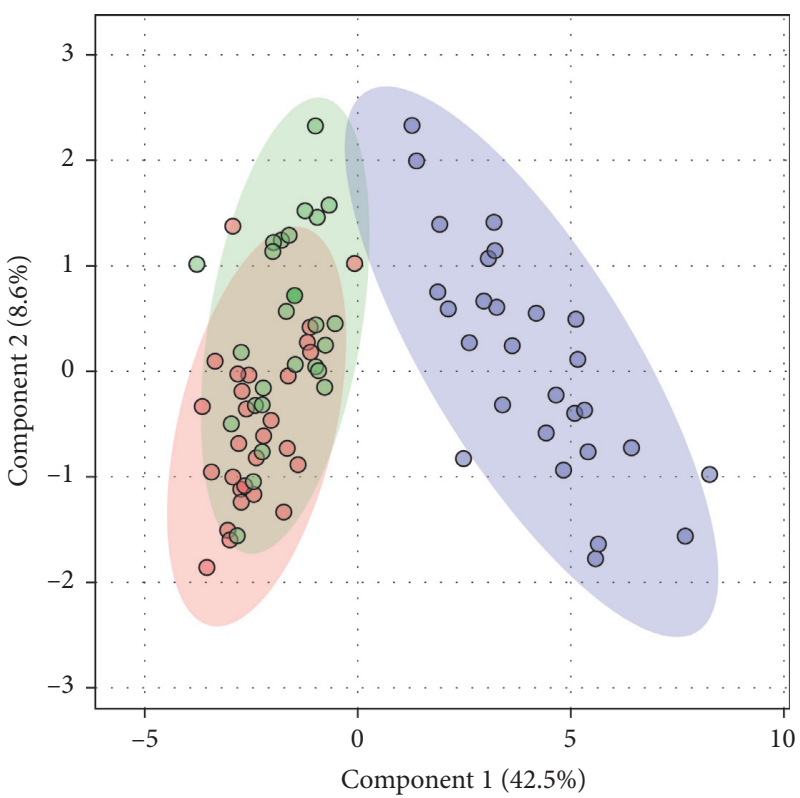

- Control

- Posttreatment

- Pretreatment

FIgURE 3: The partial least-squares discriminant analysis recognition based on the follicular fluid metabolomic profiling.

number of follicles [42]. Testosterone can improve the ovarian microenvironment and increase the expression of connexin 37 ( $\mathrm{Cx} 37)$ to promote ovarian response [43]. Low basal testosterone level is an important risk factor for low oocyte production after ovarian stimulation, which may affect the pregnancy rate of in vitro fertilization [44].

Luteal hormone is a hormone secreted by the ovary, which can inhibit the proliferation of granulosa cells induced by epidermal growth factor. It affects cell oxidative stress [45]. 8-Hydroxyguanosine (8-OHdG) and 4hydroxynonenal (4-HNE) are the most common markers of oxidative stress. The increase in luteal hormone, 8OHdG, and 4-HNE could cause the increase in oxidative stress response. Oxidative stress can cause enzyme inhibition, protein synthesis inhibition, DNA/RNA synthesis inhibition, and so on, which may be factors affecting oocyte 


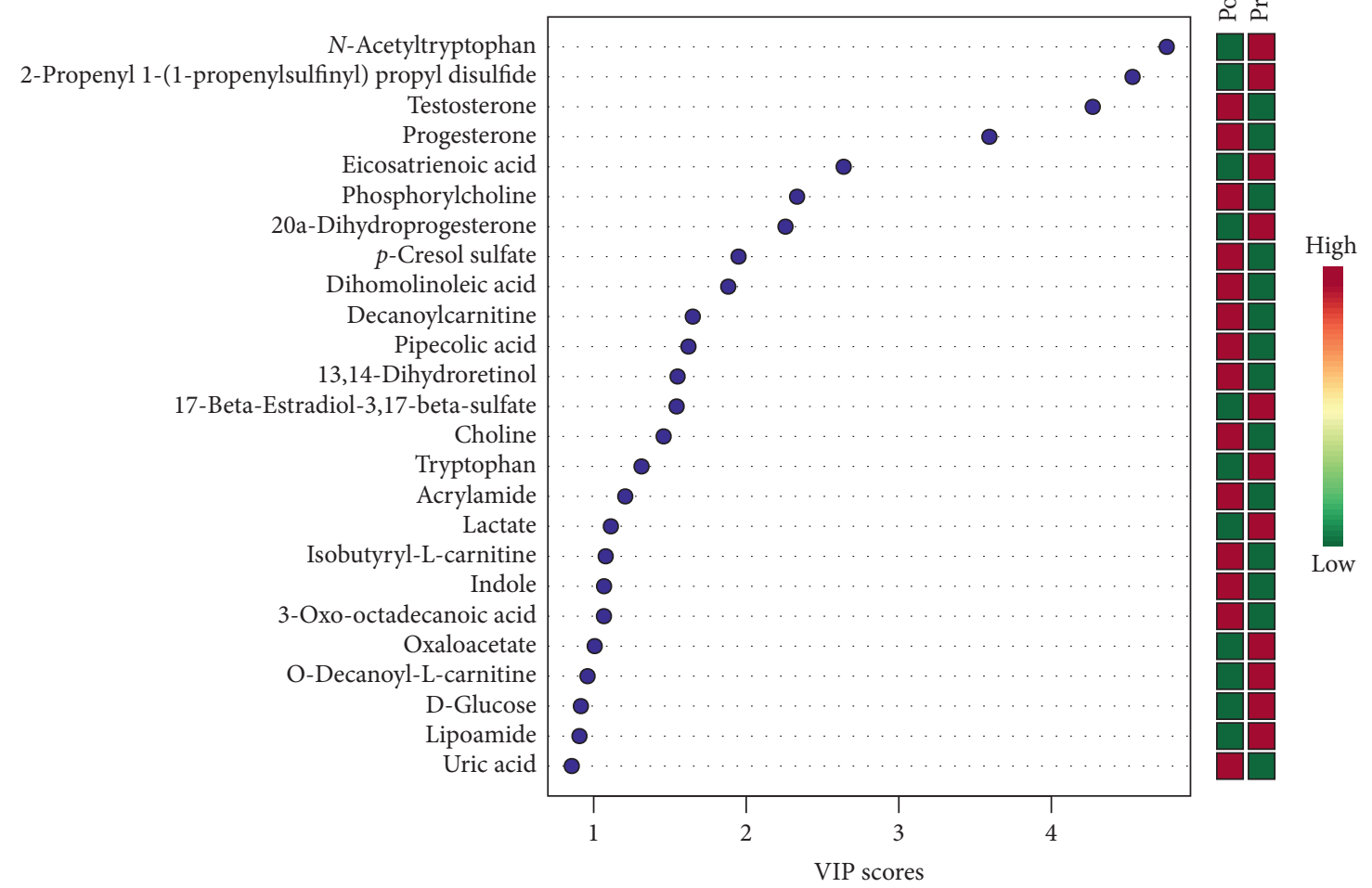

Figure 4: The VIP scores of each potential biomarker to the discrimination between the pretreatment group and the posttreatment group.

quality [46]. Luteal hormone can also affect energy metabolism. Mitochondria are the factories of cell energy, which produce a lot of ATP to activate cell activity in aerobic respiration, and participate in steroid production and cell aging. Xu et al. found that the number of abnormal mitochondria of granulosa cells in patients with endometriosis was too large [47]. Cell aging is mainly due to the reduction of oxidative phosphorylation and ATP production [48]. Mitochondrial dysfunction can lead to spindle defects and chromosomal diseases of oocytes and affect the development, maturation, and fertilization of oocytes. The high level of luteal hormone and the decrease in the ATP level lead to the decrease in oocyte quality. The high level of luteinizing hormone will also cause the decrease in carnitine levels [31]. Carnitine can protect oocytes and improve the quality of oocytes. The increase in luteinizing hormone level will cause the decrease in oocyte quality and low fertilization rate. $17 \beta$-estradiol- 3 and $17 \beta$ sulfate are the metabolites of estrogen. Estradiol is closely related to the fertilization ability of oocytes, and its metabolites could stimulate the production of progesterone. However, if the level of estradiol is too high, it can destroy the blood flow of uterus and ovary and may lead to anoxia of follicles to damage the development potential of embryos. Jiang et al. found that women with high estradiol levels had poor pregnancy outcomes [49]. In our study, there were significant differences in the number of oocytes obtained, the number of transplantable embryos, and IVF fertilization rate between the posttreatment group and the pretreatment group $(p<0.05)$.

Tryptophan may affect oocyte development and follicle quality through immunity. It can be transformed into melatonin. Melatonin can delay the aging of mouse oocytes after ovulation through SIRT1 MnSOD-dependent pathway [50], which can improve the inhibition of bisphenol A (BPA) on the meiosis and fertilization of oocytes to improve the quality of oocytes [51]. Tumor necrosis factor- $\alpha$ (TNF) is a kind of cytokine, which is produced not only by various cells in the immune system but also by various cells in the reproductive system. Indole is produced by the metabolism of tryptophan [52]. Indole may reduce TNF-mediated NF-KB activation. Oocytes, granulosa cells, and stromal cells are important sources of TNF, which can reduce the number of oocytes and primordial follicles by stimulating oocyte apoptosis. Therefore, TNF can be used as an important ovarian factor to determine the size of primordial follicle pool [53]. The absence of TNF receptors (tnfrsflb) leads to the obvious acceleration of follicle growth, which is an important mediator of TNF function in the ovary and an important regulator of follicular development [54].

13,14-Dihydroretinol is related to retinoic acid metabolism. Retinoic acid is the intermediate metabolite of vitamin A. Some studies have found that vitamin A can protect ROS-induced cell oxidative stress [55]. 13,14-Dihydroretinol was upregulated after the intervention of $\mathrm{LWDH}$ pill to affect oocyte development and oocyte quality. 


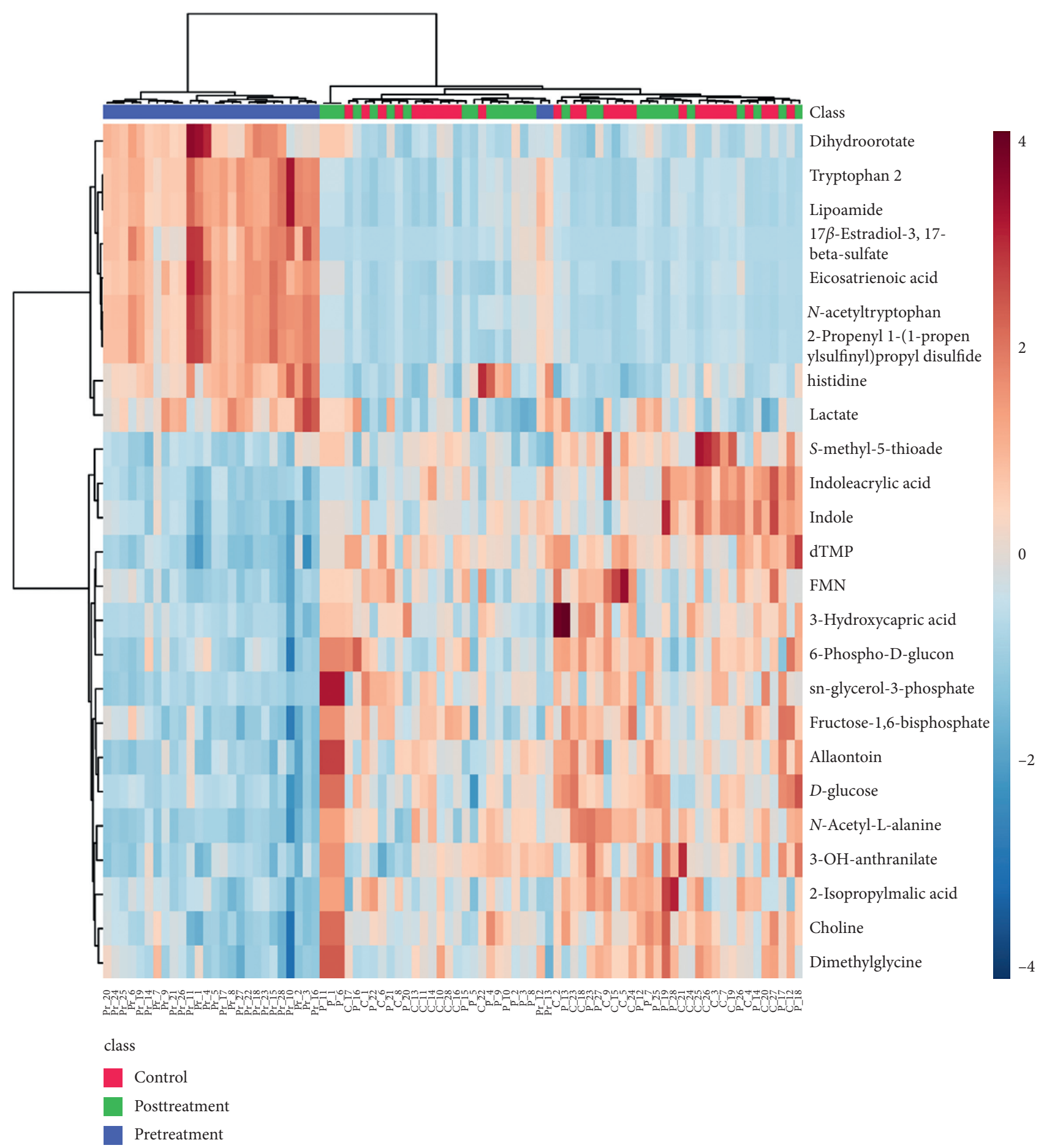

FIgURE 5: The hierarchical clustering heatmap of the potential biomarkers.

Acrylamide has toxic effects on the thyroid, red blood cells, skeletal muscle, smooth muscle, and nervous system. At the same time, it will affect the proliferation of mouse granulosa cells and the production of progesterone [56], resulting in the early apoptosis of oocytes [57]. p-Cresol sulfate is a biomarker of renal decline [58]. Some studies have shown that mitochondrial damage is one of the main pathological mechanisms of uremic poisoning [59]. Mitochondria, as the biological signal conduit of apoptosis, necrosis, and autophagy, affects oocyte quality and apoptosis. 5,8,11-Eicosapentaenoic is produced from arachidonic acid by cytochrome P450 (CYP) cyclooxygenase, which may be involved in lipid metabolism. The specific mechanism is unclear.

Metabolic changes are relevant to many diseases. In previous study, lipidomics has contributed an improved understanding of a potential underlying mechanism of ovarian aging and poor oocyte quality. Lipids seem to play a 


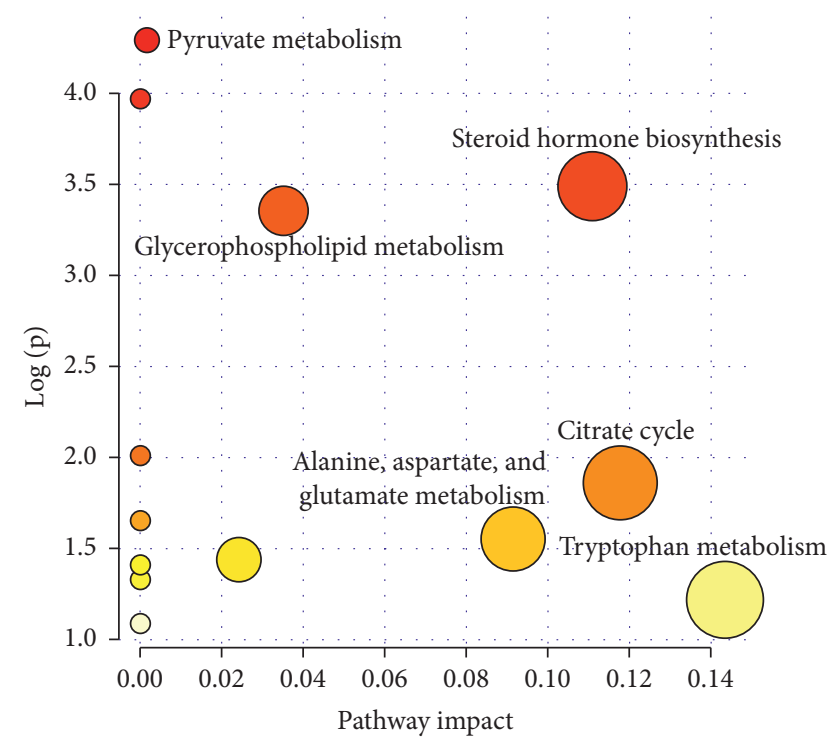

FIGURE 6: The metabolic pathways related to DOR, as analyzed by MetaboAnalyst.

crucial role in ovary aging, and further analysis and validation of potential lipid biomarkers may facilitate improved IVF in the future [60]. In our study, a group of biomarkers were found and used to characterize disease based on metabolomics. It could be helpful to understand the key features of DOR and be useful for the prevention, diagnosis, and treatment of DOR.

\section{Conclusions}

A pseudotargeted metabolomics profiling on the follicular fluid was successfully established and integrally investigated. The follicular fluid metabolome was obviously altered in DOR patients after LWDH pill treatment. After the pseudotarget metabolomics screening, a total of 21 metabolic biomarker candidates, which may be related to DOR were characterized, and their correlative metabolomic pathways were revealed. In addition, it was speculated that the potential active targets of LWDH pill on DOR may be more comprehensive than chemical treatments. The study demonstrated that the metabolomics strategy might serve as a powerful approach for investigating the mechanisms of DOR and LWDH pills using the processing method, while providing solutions to explore the molecular basis of diseases and identify potential biomarkers.

\section{Data Availability}

The datasets analyzed during the current study are available from the corresponding author on reasonable request.

\section{Disclosure}

Jimei Xiao and Jingyan Song are the co-first authors. There has been no significant financial support for this work that could have influenced its outcome.

\section{Conflicts of Interest}

The authors declare that there are no conflicts of interest associated with this publication.

\section{Authors' Contributions}

Zhengao Sun and Jingyan Song articipated in the design of this study. Jimei Xiao and Yuanhong Sa carried out the concepts, design, and definition of intellectual content. Lihua Yuan and Jiayin Guo carried out data analysis and manuscript preparation.

\section{Acknowledgments}

This work was supported by the National Natural Science Foundation of China (no. 81674018).

\section{References}

[1] F. I. Sharara, R. T. Scott, and D. B. Seifer, "The detection of diminished ovarian reserve in infertile women," American Journal of Obstetrics and Gynecology, vol. 179, no. 3, pp. 804-812, 1998.

[2] K. Devine, S. L. Mumford, M. Wu, A. H. DeCherney, M. J. Hill, and A. Propst, "Diminished ovarian reserve in the United States assisted reproductive technology population: diagnostic trends among 181, 536 cycles from the Society for Assisted Reproductive Technology Clinic Outcomes Reporting System," Fertility and Sterility, vol. 104, no. 3, pp. 612-619, 2015.

[3] Q. Liang, Y. Cao, and Q. Chen, "Research progress of Liuwei Dihuang pill treatment of premature ovarian failure," Medical Recapitulate, vol. 25, no. 17, pp. 3508-3512, 2019.

[4] Y. Luo, "Effect of LiuweiDihuang pills on ovarian function in natural aging SD rats," Journal of Ningxia Medical University, vol. 40, no. 5, pp. 512-516, 2018.

[5] J. Wang and G. Xia, "Xia Guicheng's experience in treating premature ovarian insufficiency by "Heart-kidney-uterus Axis" theory," Journal of Traditional Chinese Medicine, vol. 59, no. 7, pp. 554-557, 2018.

[6] M. J. Bertoldo, L. Nadal-Desbarats, N. Gérard, A. Dubois, P. K. Holyoake, and C. G. Grupen, "Differences in the metabolomic signatures of porcine follicular fluid collected from environments associated with good and poor oocyte quality," Reproduction, vol. 146, no. 3, pp. 221-231, 2013.

[7] T. T. Y. Chiu, M. S. Rogers, E. L. K. Law, C. M. Briton-Jones, L. P. Cheung, and C. J. Haines, "Follicular fluid and serum concentrations of myo-inositol in patients undergoing IVF: relationship with oocyte quality," Human Reproduction, vol. 17, no. 6, pp. 1591-1596, 2002.

[8] A. Zhang, Q. Liu, Z. Hongwei et al., "Phenotypic characterization of nanshi oral liquid alters metabolic signatures during disease prevention," Scientific Reports, vol. 6, Article ID 19333, 2016.

[9] H. Chu, A. Zhang, Y. Han et al., "Metabolomics approach to explore the effects of Kai-Xin-San on Alzheimer's disease using UPLC/ESI-Q-TOF mass spectrometry," Journal of Chromatography B, vol. 1015, pp. 50-61, 2016.

[10] L. Lionetto, C. Matilde, B. Elisa et al., "The omics in migraine," The Journal of Headache and Pain, vol. 14, no. 55, 2013.

[11] Y. Nan, X. Zhou, Q. Liu et al., "Serum metabolomics strategy for understanding pharmacological effects of ShenQi pill 
acting on kidney yang deficiency syndrome," Journal of Chromatography B, vol. 1026, pp. 217-226, 2016.

[12] H. Zha, Y. Cai, Y. Yin, Z. Wang, K. Li, and Z.-J. Zhu, "SWATHtoMRM: development of high-coverage targeted metabolomics method using SWATH technology for biomarker discovery," Analytical Chemistry, vol. 90, no. 6, pp. 4062-4070, 2018.

[13] R. Liu, X. Hu, X. Zhang et al., "Metabolomics strategy using high resolution mass spectrometry reveals novel biomarkers and pain-relief effect of traditional Chinese medicine prescription Wu-Zhu-Yu decoction acting on headache modelling rats," Molecules, vol. 22, no. 12, 2017.

[14] S. Chen, H. Kong, X. Lu et al., "Pseudotargeted metabolomics method and its application in serum biomarker discovery for hepatocellular carcinoma based on ultra high-performance liquid chromatography/triple quadrupole mass spectrometry," Analytical Chemistry, vol. 85, no. 17, pp. 8326-8333, 2013.

[15] L. Wang, B. Su, Z. Zeng et al., "Ion-pair selection method for pseudotargeted metabolomics based on SWATH MS acquisition and its application in differential metabolite discovery of type 2 diabetes," Analytical Chemistry, vol. 90, no. 19, pp. 11401-11408, 2018.

[16] P. Luo, P. Yin, W. Zhang et al., "Optimization of large-scale pseudotargeted metabolomics method based on liquid chromatography-mass spectrometry," Journal of Chromatography A, vol. 1437, pp. 127-136, 2016.

[17] G. Nyamundanda, I. Gormley, Y. Fan, W. M. Gallagher, and L. Brennan, "MetSizeR: selecting the optimal sample size for metabolomic studies using an analysis based approach," $B M C$ Bioinformatics, vol. 14, no. 1, p. 338, 2013.

[18] Association, "Expert consensus on hormone replacement therapy for premature ovarian insufficiency," Chinese Journal of Obstetrics and Gynecology, vol. 51, no. 12, pp. 881-886, 2016.

[19] J. Song, X. Wang, G. Ying et al., "Novel high-coverage targeted metabolomics method (SWATHtoMRM) for exploring follicular fluid metabolome alterations in women with recurrent spontaneous abortion undergoing in vitro fertilization," Scientific Reports, vol. 9, no. 1, p. 10873, 2019.

[20] J. Chong, D. S. Wishart, and J. Xia, "Using MetaboAnalyst 4.0 for comprehensive and integrative metabolomics data analysis," Current Protocols in Bioinformatics, vol. 68, no. 1, Article ID e86, 2019.

[21] W. H. Meck, R. A. Smith, and C. L. Williams, "Organizational changes in cholinergic activity and enhanced visuospatial memory as a function of choline administered prenatally or postnatally or both," Behavioral Neuroscience, vol. 103, no. 6, pp. 1234-1241, 1989.

[22] S. H. Zeisel and M. D. Niculescu, "Perinatal choline influences brain structure and function," Nutrition Reviews, vol. 64, no. 4, pp. 197-203, 2006.

[23] X. Jiang, A. A. West, and M. A. Caudill, "Maternal choline supplementation: a nutritional approach for improving offspring health?" Trends in Endocrinology \& Metabolism, vol. 25, no. 5, pp. 263-273, 2014.

[24] J. Yan, X. Jiang, A. A. West et al., "Maternal choline intake modulates maternal and fetal biomarkers of choline metabolism in humans," The American Journal of Clinical Nutrition, vol. 95, no. 5, pp. 1060-1071, 2012.

[25] J. M. Lauder and U. B. Schambra, "Morphogenetic roles of acetylcholine," Environmental Health Perspectives, vol. 107, no. 1, pp. 65-69, 1999.

[26] J. K. Blusztajn and T. J. Mellott, "Choline nutrition programs brain development via DNA and histone methylation,"
Central Nervous System Agents in Medicinal Chemistry, vol. 12, no. 2, pp. 82-94, 2012.

[27] K. Bender, S. Walsh, A. C. O. Evans, T. Fair, and L. Brennan, "Metabolite concentrations in follicular fluid may explain differences in fertility between heifers and lactating cows," Reproduction, vol. 139, no. 6, pp. 1047-1055, 2010.

[28] A. Veshkini, A. A. Khadem, A. Mohammadi-Sangcheshmeh, A. A. Alamouti, M. Soleimani, and E. L. Gastal, "Linolenic acid improves oocyte developmental competence and decreases apoptosis ofin vitro-produced blastocysts in goat," Zygote, vol. 24, no. 4, pp. 537-548, 2016.

[29] F. M. Vaz and R. J. Wanders, "Carnitine biosynthesis in mammals," Biochem Journal, vol. 361, no. 3, pp. 417-429, 2002.

[30] H.-Y. Xu, X.-G. Yang, S.-S. Lu et al., "Treatment with acetyl-1carnitine during in vitro maturation of buffalo oocytes improves oocyte quality and subsequent embryonic development," Theriogenology, vol. 118, pp. 80-89, 2018.

[31] Z. Zare, R. Masteri Farahani, M. Salehi et al., "Effect of L-carnitine supplementation on maturation and early embryo development of immature mouse oocytes selected by brilliant cresyle blue staining," Journal of Assisted Reproduction and Genetics, vol. 32, no. 4, pp. 635-643, 2015.

[32] G.-Q. Wu, B.-Y. Jia, J.-J. Li et al., "L-carnitine enhances oocyte maturation and development of parthenogenetic embryos in pigs," Theriogenology, vol. 76, no. 5, pp. 785-793, 2011.

[33] K. R. Dunning and R. L. Robker, "Promoting lipid utilization with l-carnitine to improve oocyte quality," Animal Reproduction Science, vol. 134, no. 1-2, pp. 69-75, 2012.

[34] M. Fathi and K. H. El-Shahat, "L-carnitine enhances oocyte maturation and improves in vitro development of embryos in dromedary camels (Camelus dromedaries)," Theriogenology, vol. 104, pp. 18-22, 2017.

[35] J. You, J. Lee, S.-H. Hyun, and E. Lee, "L-carnitine treatment during oocyte maturation improves in vitro development of cloned pig embryos by influencing intracellular glutathione synthesis and embryonic gene expression," Theriogenology, vol. 78, no. 2, pp. 235-243, 2012.

[36] A. Jafari, S. Dashti-Khavidaki, H. Khalili, and M. LessanPezeshki, "Potential nephroprotective effects ofl-carnitine against drug-induced nephropathy: a review of literature," Expert Opinion on Drug Safety, vol. 12, no. 4, pp. 523-543, 2013.

[37] B. Yuan, L. Shuang, J.-W. Kwon et al., "The role of glucose metabolism on porcine oocyte cytoplasmic maturation and its possible mechanisms," PLoS One, vol. 11, no. 12, Article ID e0168329, 2016.

[38] M. L. Sutton-McDowall, R. B. Gilchrist, and J. G. Thompson, "The pivotal role of glucose metabolism in determining oocyte developmental competence," Reproduction, vol. 139, no. 4, pp. 685-695, 2010.

[39] J.-L. Yang, C.-P. Zhang, L. Li et al., "Testosterone induces redistribution of forkhead box-3a and down-regulation of growth and differentiation factor 9 messenger ribonucleic acid expression at early stage of mouse folliculogenesis," Endocrinology, vol. 151, no. 2, pp. 774-782, 2010.

[40] S. J. Weil, K. Vendola, J. Zhou et al., “Androgen receptor gene expression in the primate ovary: cellular localization, regulation, and functional correlations," The Journal of Clinical Endocrinology \& Metabolism, vol. 83, no. 7, pp. 2479-2485, 1998.

[41] T. E. Hickey, D. L. Marrocco, R. B. Gilchrist, R. J. Norman, and D. T. Armstrong, "Interactions between androgen and growth factors in granulosa cell subtypes of porcine antral 
Follicles1," Biology of Reproduction, vol. 71, no. 1, pp. 45-52, 2004.

[42] H. Cardenas, J. R. Herrick, and W. F. Pope, "Increased ovulation rate in gilts treated with dihydrotestosterone," Reproduction, vol. 123, no. 4, pp. 527-533, 2002.

[43] Y. Zhang, X. Yang, Y. Kuai et al., "Effect of testosterone on the Connexin37 of sexual mature mouse cumulus oocyte complex," Journal of Ovarian Research, vol. 9, no. 1, p. 82, 2016.

[44] J. Guo, Q. Zhang, Y. Li, W. Wang, and D. Yang, "Low level of basal testosterone: a significant risk factor for poor oocyte yield after ovulation induction," Reproduction, Fertility and Development, vol. 28, no. 3, pp. 286-292, 2016.

[45] Y. Zhu, X. Huang, and J. Shao, "Clinical pharmacology of progestin," World Clinical Drugs, vol. 35, no. 11, pp. 641-645, 2014.

[46] L. L. Wu, C. C. Chiou, P. Y. Chang et al., "Urinary 8-OHdG: a marker of oxidative stress to DNA and a risk factor for cancer, atherosclerosis and diabetics," Clinica Chimica Acta, vol. 339, no. 1-2, pp. 1-9, 2004.

[47] B. Xu, G. Nan, S. Wei et al., "Oocyte quality is decreased in women with minimal or mild endometriosis," Scientific Reports, vol. 5, Article ID 10779, 2015.

[48] A. Ben-Meir, C. Maggi, Y. Wang et al., "Coenzyme Q10 restores oocyte mitochondrial function and fertility during reproductive aging," Aging Cell, vol. 14, no. 5, pp. 887-895, 2015.

[49] Z. Jiang, L. Jin, W. Shi et al., "A combination of follicle stimulating hormone, estradiol and age is associated with the pregnancy outcome for women undergoing assisted reproduction: a retrospective cohort analysis," Science China Life Sciences, vol. 62, no. 1, pp. 112-118, 2019.

[50] Q. Yang, S. Dai, X. Luo et al., "Melatonin attenuates postovulatory oocyte dysfunction by regulating SIRT1 expression," Reproduction, vol. 156, no. 1, pp. 81-92, 2018.

[51] M. Zhang, X. Bo, C. Zhou et al., "Melatonin protects oocyte quality from Bisphenol A-induced deterioration in the mouse," Journal of Pineal Research, vol. 62, no. 3, 2017.

[52] G. V. Sridharan, C. Wu, K. Choi et al., "Prediction and quantification of bioactive microbiota metabolites in the mouse gut," Nature Communications, vol. 5, p. 5492, 2014.

[53] J. L. Marcinkiewicz, S. K. Balchak, and L. J. Morrison, "The involvement of tumor necrosis factor-a (TNF) as an intraovarian regulator of oocyte apoptosis in the neonatal rat," Frontiers in Bioscience, vol. 7, no. 1-3, Article ID d1997, 2002.

[54] C. R. Greenfeld, K. F. Roby, M. E. Pepling, J. K. Babus, P. F. Terranova, and J. A. Flaws, "Tumor necrosis factor (TNF) receptor type 2 is an important mediator of TNF alpha function in the mouse Ovaryl," Biology of Reproduction, vol. 76, no. 2, pp. 224-231, 2007.

[55] M. O. Özkaya and M. Nazıroğlu, "Multivitamin and mineral supplementation modulates oxidative stress and antioxidant vitamin levels in serum and follicular fluid of women undergoing in vitro fertilization," Fertility and Sterility, vol. 94, no. 6, pp. 2465-2466, 2010.

[56] R. P. Webster, V. H. J. Roberts, and L. Myatt, "Protein nitration in placenta-functional significance," Placenta, vol. 29, no. 12, pp. 985-994, 2008.

[57] A. Agarwal, S. Gupta, L. Sekhon, and R. Shah, "Redox considerations in female reproductive function and assisted reproduction: from molecular mechanisms to health implications," Antioxidants \& Redox Signaling, vol. 10, no. 8, pp. 1375-1404, 2008.
[58] C. Barrios, P. Tess, V. Judith et al., "Gut-Microbiota-metabolite Axis in early renal function decline," PLoS One, vol. 10, no. 8, Article ID e0134311, 2015.

[59] C.-Y. Sun, M.-L. Cheng, H.-C. Pan, J.-H. Lee, and C.-C. Lee, "Protein-bound uremic toxins impaired mitochondrial dynamics and functions," Oncotarget, vol. 8, no. 44, pp. 77722-77733, 2017.

[60] F. B. Cordeiro, D. A. Montani, E. J. Pilau, F. C. Gozzo, R. Fraietta, and E. G. L. Turco, "Ovarian environment aging: follicular fluid lipidomic and related metabolic pathways," Journal of Assisted Reproduction and Genetics, vol. 35, no. 8, pp. 1385-1393, 2018. 